\title{
FITOTERAPIA
}

\section{Phytochemical communication}

\section{Constituents of Caesalpinia pyramidalis}

\author{
C.C. Mendes ${ }^{\mathrm{a}}$, M.V. Bahia ${ }^{\mathrm{a}}$, J.M. David ${ }^{\mathrm{a}, *}$, J.P. David $^{\mathrm{b}}$ \\ ${ }^{a}$ Instituto de Química, Universidade Federal da Bahia, Campus Ondina, 40170-290, Salvador-BA, \\ Brazil \\ ${ }^{\mathrm{b}}$ Faculdade de Farmácia, Universidade Federal da Bahia, Campus Ondina, 40170-290, \\ Salvador-BA, Brazil
}

Received 19 July 1999; accepted 13 September 1999

\begin{abstract}
Two new glycosyl phenylpropenoid acids, 4-O- $\beta$-glucopyranosyloxy-( $Z$ )-7-hydroxycinnamic acid (1) and 4-O- $\beta$-glucopyranosyloxy-(Z)-8-hydroxycinnamic acid (2), besides lupeol and aghatisflavone, were isolated from the leaves of Caesalpinia pyramidalis. (C) 2000 Elsevier Science B.V. All rights reserved.
\end{abstract}

Keywords: Caesalpinia pyramidalis; Phenylpropenoid glycosides; Biflavones; Triterpenes

Plant. Caesalpinia pyramidalis Tul. (Caesalpinaceae), dried leaves, collected in Valente (BA), Brazil by Prof. W.A. Lopes, in August 1993, and identified by Prof. L. Scardino (IB-UFBA). Voucher specimen is deposited under number 0240291 at Herbário Alexandre Leal Costa of Instituto de Biologia of Universidade Federal da Bahia.

Uses in traditional medicine. For fever and stomach diseases, and as diuretic [1].

Previously isolated constituents. No report.

New-isolated constituents. 4-O- $\beta$-Glucopyranosyloxy-( $Z$ )-7-hydroxycinnamic acid (1, yield $0.001 \%)$, 4-O- $\beta$-glucopyranosyloxy- $(Z)-8$-hydroxycinnamic acid (2) [2]

\footnotetext{
* Corresponding author.

E-mail address: jmdavid@ufba.br (J.M. David) 
(0.00073), isolated as acetyl derivatives 1a and 2a, respectively; lupeol [3] (0.034) and aghatisflavone [4] (0.076).

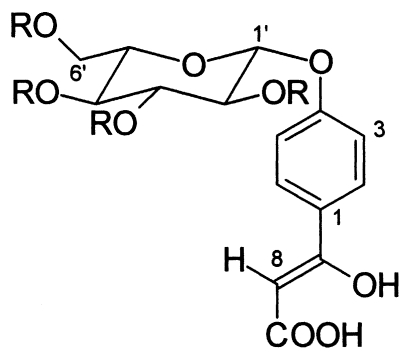

$1 \mathrm{R}=\mathrm{H}$

1a $\mathrm{R}=\mathrm{COCH}_{3}$

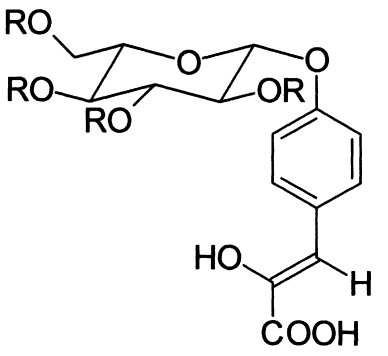

$2 \mathrm{R}=\mathrm{H}$ 2a $\mathrm{R}=\mathrm{COCH}_{3}$

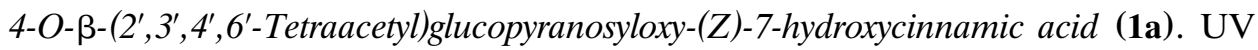
$\max (\mathrm{MeOH}): 218$ ( $\varepsilon$ 61963), 274 (39840); $\left(+\mathrm{AlCl}_{3}\right): 222$ (61868), 282 (36409), 330 (20767); $\left(+\mathrm{AlCl}_{3}+\mathrm{HCl}\right): 224$ (60033), 282 (35331), 330 (20818) nm; IR bands (KBr): 3415, 2953, 1754, 1658, 1228, $846 \mathrm{~cm}^{-1} ;{ }^{1} \mathrm{H}-\mathrm{NMR}\left(300 \mathrm{MHz}, \mathrm{CDCl}_{3}\right): \delta 7.79$ $(2 \mathrm{H}, d, J 8.5 \mathrm{~Hz}, \mathrm{H}-2$ and H-6), $7.04(2 \mathrm{H}, d, J 8.5 \mathrm{~Hz}, \mathrm{H}-3$ and $\mathrm{H}-5), 6.57(1 \mathrm{H}, s$, $\mathrm{H}-8), 6.39\left(1 \mathrm{H}, d, J 8.7 \mathrm{~Hz}, \mathrm{H}-1^{\prime}\right), 5.68-4.02\left(4 \mathrm{H}, m, \mathrm{H}-2^{\prime}-\mathrm{H}-5^{\prime}\right), 4.38\left(1 \mathrm{H}, d d, J_{1}\right.$ 12.5, $\left.J_{2} 2.8, \mathrm{H}-6^{\prime}\right), 4.22\left(1 \mathrm{H}, d d, J_{1} 12.5, J_{2} 2.0, \mathrm{H}-6^{\prime}\right), 2.11\left(6 \mathrm{H}, s, \underline{\mathrm{C}}_{3} \mathrm{CO}\right), 2.04$ $\left.6 \mathrm{H}, s, \underline{\mathrm{CH}}_{3} \mathrm{CO}\right), 12.81(1 \mathrm{H}, s, \mathrm{O} \overline{\mathrm{H}}) ;{ }^{13} \mathrm{C}-\mathrm{NMR}\left(75 \mathrm{MHz}, \mathrm{CDCl}_{3}\right): 123.41(\mathrm{C}-1)$, 128.14 (C-2 and C-6), 116.43 (C-3 and C-5), 162.69 (C-4), 159.44 (C-7), 98.71 (C-8), 182.08 (C-9), $104.33\left(\mathrm{C}-1^{\prime}\right), 67.78,70.73,73.66$ and $73.67\left(\mathrm{C}-2^{\prime}-\mathrm{C}-5^{\prime}\right), 61.23\left(\mathrm{C}-6^{\prime}\right)$, 19.87, 20.40, 20.48 and $20.50\left(4 \times \underline{\mathrm{CH}}_{3} \mathrm{CO}\right), 170.43,170,29,169.21$ and 168.40 $(4 \times \underline{\mathrm{COO}})$.

4-O- $\beta$-(2', 3', 4', 6'-Tetraacetyl)glucopyranosyloxy-(Z)-8-hydroxycinnamic acid (2a). UV $\max (\mathrm{MeOH}): 226$ ( $\varepsilon$ 42794), 270 (27380); ( $\mathrm{AlCl}_{3}$ ): 234 (87890), 280 (41487), 304 (44211); $\left(+\mathrm{AlCl}_{3}+\mathrm{HCl}\right): 234$ (88656), 280 (42324), 304 (45116) nm; IR bands (KBr): 3419, 1751, 1654, 1240, $840 \mathrm{~cm}^{-1} ;{ }^{1} \mathrm{H}-\mathrm{NMR}\left(300 \mathrm{MHz}, \mathrm{CDCl}_{3}\right): \delta 7.94(2 \mathrm{H}$, $d, J 8.4 \mathrm{~Hz}, \mathrm{H}-2$ and H-6), $7.37(2 \mathrm{H}, d, J 8.4 \mathrm{~Hz}, \mathrm{H}-3$ and $\mathrm{H}-5), 6.65(1 \mathrm{H}, s, \mathrm{H}-7)$, $6.41\left(1 \mathrm{H}, d, J 8.7 \mathrm{~Hz}, \mathrm{H}-1^{\prime}\right), 5.69-4.02\left(4 \mathrm{H}, m, \mathrm{H}-2^{\prime}-\mathrm{H}-5^{\prime}\right), 4.39\left(1 \mathrm{H}, d d, J_{1} 12.5, J_{2}\right.$ 1.5, H-6'), $4.22\left(1 \mathrm{H}, d d, J_{1} 12.5, J_{2} 1.5, \mathrm{H}-6^{\prime}\right), 2.12\left(6 \mathrm{H}, s, \underline{\mathrm{C}}_{3} \mathrm{CO}\right), 2.04(6 \mathrm{H}, s$, $\left.\underline{\mathrm{CH}}_{3} \mathrm{CO}\right), 12.81(1 \mathrm{H}, s, \mathrm{O} \overline{\mathrm{H}}) ;{ }^{13} \mathrm{C}-\mathrm{NMR}\left(75 \mathrm{MHz}, \mathrm{CDCl}_{3}\right.$ ): 128.69 (C-1), 127.48 (C-2 
and C-6), 122.74 (C-3 and C-5), 162.71 (C-4), 127.30 (C-7), 153.60 (C-8), 181.95 (C-9), $105.94\left(\mathrm{C}-1^{\prime}\right), 67.74,70.68,73.61$ and $73.62\left(\mathrm{C}-2^{\prime}-\mathrm{C}-5^{\prime}\right), 61.26\left(\mathrm{C}-6^{\prime}\right), 20.96$, 20.44, 20.38 and $19.91\left(4 \times \underline{\mathrm{CH}}_{3} \mathrm{CO}\right), 170.37,170.11,169.13$ and $168.67(4 \times \underline{\mathrm{COO}})$.

\section{Acknowledgements}

C.C. Mendes and M.V. Bahia are grateful to Fundação Coordenação de Aperfeiçoamento de Pessoal de Nível Superior (CAPES) and Conselho Nacional do Desenvolvimento Científico e Tecnológico (CNPq) for the fellowships. The authors also thank PADCT/CNPq and FINEP for financial support.

\section{References}

[1] Inventário de Plantas Medicinais do Estado da Bahia. Governo do Estado da Bahia (SEPLANTEC), 1979:334.

[2] Shigemori H, Sakai N, Miyoshi E, Shizuki Y, Yamamura S. Tetrahedron 1990;46:383.

[3] Mahato SB, Kundu AP. Phytochemistry 1994;37:1517.

[4] Chari VM, Ilyas M, Wagner H et al. Phytochemistry 1977;16:1273. 